\title{
Steady state multipactor and dependence on material properties
}

\author{
R. A. Kishek, Y. Y. Lau, and D. Chernin ${ }^{\text {a) }}$ \\ Department of Nuclear Engineering and Radiological Sciences, University of Michigan, Ann Arbor, \\ Michigan 48109-2104
}

(Received 2 July 1996; accepted 12 November 1996)

\begin{abstract}
The interaction of multipactor discharge and an rf circuit is analyzed with the use of a simple model, in which the multipactor electrons are in the form of a single sheet that is released from the surface with a monoenergetic velocity. An explicit formula is derived for the saturation level of multipactor current in steady state. This formula is given in terms of the secondary electron yield properties of the multipactoring surfaces and the level of the external rf drive. It is valid when the quality factor $Q$ of the rf circuit is higher than 10, in which case the space charge effects do not contribute significantly to the saturation level. When it occurs, the steady state multipactor may consume tens of percents of the external rf power that is needed to sustain the gap voltage. Numerical computations determine the accessibility to steady state from the transient buildup. In particular, they suggest various conditions for the multipactor to exhibit in a burst mode or in a steady state mode. The dynamic linkage of the rf circuit and material properties allows the construction of the susceptibility diagram for various materials, within the limitations imposed by the present model.

(C) 1997 American Institute of Physics. [S1070-664X(97)03702-6]
\end{abstract}

\section{INTRODUCTION}

Multipactor discharge is a resonant, low to medium voltage phenomenon ${ }^{1-3}$ frequently observed in microwave systems such as rf windows, ${ }^{4}$ accelerator structures, ${ }^{5}$ and rf satellite payloads. ${ }^{6}$ Multipactor is usually undesirable, since it loads the cavity, dissipates power, and causes damage to the components. It can be observed visually as a glowing line connecting the multipacting surfaces. ${ }^{7}$ Whenever a vacuum is present and the geometry, fields and surface are such that multipactor can occur, then a seed electron can trigger the discharge. Such a seed electron can come from a stray beam electron, field emission at some macroscopic irregularities on the surface, or even a cosmic ray. To understand the phenomenon, consider a microwave cavity with walls made of a material having a secondary electron yield $\delta>1$. A seed electron somewhere inside the cavity will be accelerated by the rf electric field that resides in the cavity and will eventually hit one of the walls, emitting $\delta$ electrons in the process. If, at the time of impact, the electric field points into that wall, it will accelerate these secondary electrons away from it, causing them to hit another wall and emit more electrons, called the multipactor electrons. Under certain resonant conditions, for example, when the electron transit time is equal to half the rf period, this process can be continued further, leading to exponential growth of the charge density inside the cavity, and thus growth of the multipactor current.

Very little has been published on this subject. ${ }^{1-7}$ Most theoretical analyses of multipactor concentrated on the response of a single electron to an imposed rf electric field. Analytic expressions have been derived for the phase of the emitted electron, and the range of the rf electric field in which a stable, steady state multipactor may exist. ${ }^{1,2}$ While some calculations have included the space charge effects as-

${ }^{a}$ Science Application International Corporation, McLean, Virginia 22102. sociated with the multipactor electrons, ${ }^{2,3}$ most of these calculations omit the important processes of loading and detuning of the $\mathrm{rf}$ cavities as the multipactor current grows. Recently, beam loading effects in a resonant structure have been demonstrated to cause the discharge to saturate. ${ }^{8}$ Space charge forces have been shown to be insignificant relative to beam loading effects in determining the steady state $^{9}$ when the quality factor, $Q$, of the cavity is larger than 10 .

In this paper, we use a simplified model to derive the level of multipactor current in the steady state as a function of external parameters. The route to these steady state solutions is examined in detail in simulations. For simplicity, the derivation is restricted to a first order multipactor, in which the electron's transit time across the gap is approximately $1 / 2$ of the period of the $\mathrm{rf}$ electric field. The effect of a nonzero initial velocity for the secondaries, with a monoenergetic distribution, is also examined. After scaling to geometry, the external parameters can be reduced to two: one relating to the power source, and another relating to the secondary emission properties of the materials used. The accessibility of such solutions is then examined in detail and displayed for a wide range of power levels and materials. Finally, our theory is compared to published data on multipactor.

In Sec. II, we describe the model. In Sec. III, we derive the saturation level of the multipactor current, assuming that a steady state exists. In Sec. IV, we obtain the susceptibility diagram and address the accessibility of the steady state multipactor solutions. In Sec. V, we confirm the steady state solutions with simulation results and examine them in the light of published data. Some concluding remarks are given in Sec. VI.

\section{MODEL AND RELEVANT EQUATIONS}

Our model consists of an electron sheet, of surface density $\sigma$, inside a planar gap (Fig. 1). The sheet is instantaneously located at a distance $x$ from one of the electrodes. The gap separation is $D$ and the gap voltage is $V_{g}(t)$. We 


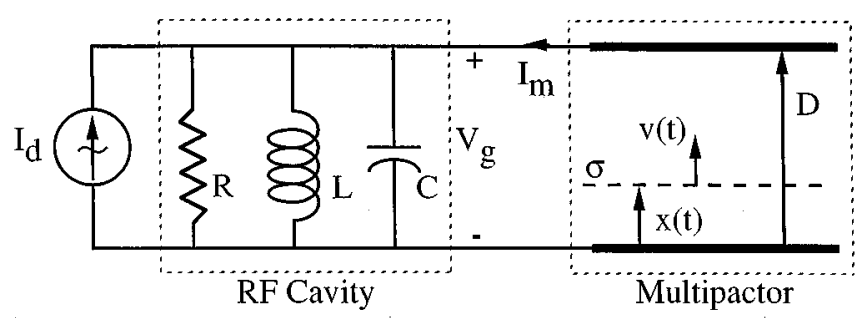

FIG. 1. Single-sheet model for interaction of multipactor with an rf cavity.

assume that the voltage $V_{g}$ that drives the multipactor is provided by an rf cavity. This cavity, with characteristic frequency $\omega_{0}$ and quality factor $Q$, is modeled by a corresponding lumped circuit with elements $R, L$, and $C$, driven by an ideal current source $I_{d}(t)$ (Fig. 1). The motion of the multipactor electron sheet induces a wall current, $I_{m}(t)$, which loads this RLC circuit. Upon impact on a gap surface, the respective incident electron sheet is removed and a new electron sheet is generated by secondary emission. The singlesheet model may be justified here on account of the newly discovered phase-focusing mechanism, ${ }^{9}$ whereby the leading multipactor electrons have a tendency to "cannibalize" the trailing ones. Briefly, the leading part of the bunch, being accelerated by the trailing part, impacts with a higher energy on a plate, therefore it produces a higher secondary yield and grows faster than the trailing part.

The evolution of $V_{g}, x$ and the multipactor current $I_{m}$ is governed by the following normalized equations: ${ }^{8}$

$$
\left(\frac{d^{2}}{d t^{2}}+\frac{1}{Q} \frac{d}{d t}+1\right) V_{g}(t)=\frac{d}{d t}\left[I_{d 0} \sin (\omega t+\phi)+I_{m}(t)\right],
$$$$
I_{m}(t)=-\sigma \frac{d x}{d t}
$$

$$
\frac{d^{2} x}{d t^{2}}=V_{g}(t)
$$

where we used the normalization scales: $D$ for distance, $\omega_{0}$ for frequency, $1 / \omega_{0}$ for time, $v=\omega_{0} D$ for velocity, $U=m v^{2}$ for energy, $U / e$ for voltage, $E=U / e D$ for electric field, and $\Sigma=\epsilon_{0} E$ for surface charge density. For example, to obtain the dimensional gap voltage (in volts), multiply the nondimensional value $V_{g}$ by $m\left(\omega_{0} D\right)^{2} / e$.

Equation (1) is the circuit equation governing the evolution of the gap voltage, driven by the normalized ideal current source $I_{d}$, (of amplitude $I_{d 0}$, frequency $\omega$, and phase $\phi$ at time $t=0)$ and by the multipactor current $I_{m}$ (Fig. 1). Note that the term $I_{m}$ in Eq. (1) is solely responsible for the nonlinear beam loading and frequency detuning of the cavity by the multipactor. It is simply the wall current induced by the motion of the sheet as given by Eq. (2), where $\sigma$ is always positive by convention. In the absence of multipactor $\left(I_{m}=0\right)$, Eq. (1) yields a sinusoidal steady state gap voltage with amplitude $r=Q I_{d 0}$ when $\omega=1$, i.e., resonantly driven. Equation (3) is the force law that governs the motion of the electron sheet, where only the force due to the gap voltage has been taken into account. Space charge forces have been

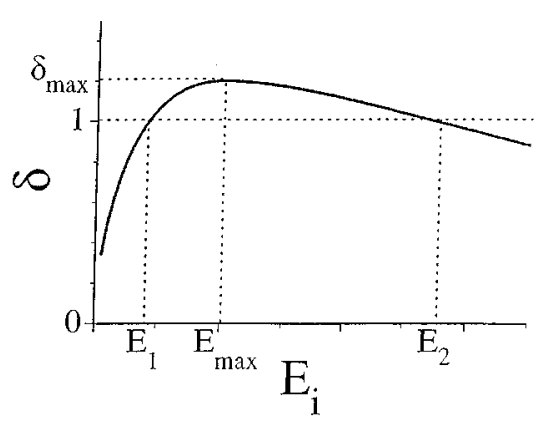

FIG. 2. Secondary electron yield, $\delta$, as a function of impact energy, $E_{i}$.

shown not to significantly affect the steady state behavior of the multipactor, ${ }^{8,9}$ especially in high $Q$ cavities ( $Q$ higher than 10, see Table II below).

On impact with a plate at time $t_{i}$, the incident electron sheet is removed and a new sheet of charge is released by secondary emission. The post-impact surface charge density $\sigma\left(t_{i}^{+}\right)$is related to the pre-impact charge density $\sigma\left(t_{i}^{-}\right)$by

$$
\sigma\left(t_{i}^{+}\right)=\delta \cdot \sigma\left(t_{i}^{-}\right),
$$

where $\delta$ is the coefficient of secondary emission which depends on the electron impact energy, $E_{i}$, of the impacting sheet. Here, $E_{i}=(d x / d t)^{2} / 2$, evaluated at $t=t_{i}^{-}$. For simplicity, we assume that the secondary electrons are emitted with a fixed initial velocity. ${ }^{10}$ In addition, we adopt Vaughan's empirical formula ${ }^{11}$ which gives the secondary yield curve plotted in Fig. 2. This shows that $\delta=1$ at two values of impact energies, $E_{1}$ and $E_{2}$, and $\delta>1$ in between. The lower energy $E_{1}$ is designated as the "first crossover point." With a single electron sheet, the steady state obtained by beam loading has been shown ${ }^{8}$ to occur at the first crossover point (i.e., $\delta=1$ and $E_{i}=E_{1}$ ).

Note that to keep our model manageable, we neglect some important surface effects that may affect the gain through the secondary electron emission process. For example, we assume all secondaries are emitted normal to the surface, whereas, in reality, they are emitted with a spread in emission angles. ${ }^{11}$ In addition to a change in the impact phase, some electrons emitted at large angles from the normal may be lost. We also neglect the effect of heating of the surface by the impinging electrons. Such heating may change the secondary electron yield and result in energy losses. These effects should be addressed in a complete analysis.

\section{THE STEADY STATE MULTIPACTOR}

Assuming the system to be in the steady state allows us to simplify the above equations sufficiently to reach an explicit solution for the multipactor current, valid for $Q>10$ according to the present model. Under steady state conditions, the charge density, $\sigma$, on the single sheet is constant for all times, implying the secondary emission coefficient at the wall is unity. A simple argument further imposes that the impact energy be equal to the first crossover point, $E_{1}$, in the secondary electron emission function. In addition, we still need to impose the resonance condition on the discharge, 
namely, impact with the second surface has to occur at half an rf cycle for a steady state first-order multipactor.

Remembering $\sigma$ is a constant, we substitute Eq. (2) into Eq. (1) and use Eq. (3) to obtain a second-order differential equation for the steady state gap voltage, $V_{g}(t)$ [see Eq. (A1) of the Appendix]. The steady state solution to $V_{g}$ includes four unknown quantities: the charge density, $\sigma$, the relative phase of the discharge to the rf, $\phi$, and the two constants of integration. To get the constants of integration we impose the continuity of $V_{g}$ and the jump in its derivative at the time of impact. Next, integrating the force law once, then twice, and imposing initial conditions $\left[x(0)=0\right.$ and $\left.v(0)=v_{0}\right]$, we obtain expressions for the velocity and location, respectively, of the sheet as a function of time and gap voltage. Imposing the conditions at impact $\left(t_{i}=\pi\right)$, namely, $x_{i}=1$, and impact velocity $v_{i}=\sqrt{2 E_{1}}$, results in two conditions on the steady state multipactor. These conditions determine the unknown quantities, in particular $\sigma$, in the steady state. Note that $\sigma v_{i}$ is a measure of the steady state multipactor current.

Unfortunately, although the above procedure can be used to obtain numerical solutions to check simulation results, it does not result in a closed-form solution, mainly because the gap voltage $V_{g}$ contains all harmonics of $\omega$. However, an excellent analytic approximation can be made when one recognizes that the gap voltage does not change appreciably during the evolution of a multipactor discharge, even if the loading level is high $(Q=1000) .{ }^{8}$ Hence we can use the first term of a Fourier series to approximate the voltage, as well as all other quantities. The Appendix outlines this procedure for the case of a first-order multipactor (higher orders introduce additional terms in the series complicating the solution), driven at resonance $(\omega=1)$, without any space charge forces.

The derivation results in a quadratic equation relating the steady state multipactor charge density $\sigma_{\text {sat }}$, to the drive current $I_{d 0}$, the impact velocity $\mu=\sqrt{2 E_{1}}$ at the first crossover point (Fig. 2), and the initial velocity $v_{0}$ of secondaries, the last two being properties of the wall material. The solution is (see the Appendix)

$$
s=\frac{-B+\sqrt{B^{2}-4 A C}}{2 A},
$$

where

$$
\begin{aligned}
& A=\frac{1}{4}\left(\mu-v_{0}\right)^{2}+\left[\frac{1}{2}-\left(\frac{\pi}{4}-\frac{2}{\pi}\right)\left(\mu+v_{0}\right)\right]^{2}, \\
& B=\frac{2}{\pi}\left(\mu^{2}-v_{0}^{2}\right), \\
& C=\left[\frac{1}{2}-\frac{\pi}{4}\left(\mu+v_{0}\right)\right]^{2}+\frac{1}{4}\left(\mu-v_{0}\right)^{2}-r^{2} .
\end{aligned}
$$

Here, $s \equiv Q \sigma_{\text {sat }}$, the steady state charge density, and $r \equiv Q I_{d 0}$, the steady state amplitude of the gap voltage in the absence of multipactor.

Note that Eq. (5) contains no explicit dependence on the quality factor of the cavity, $Q$, meaning that for a fixed amplitude of the gap voltage $r$, the steady state charge density, $\sigma_{\text {sat }}$, scales as $1 / Q$. (Thus we also see that a high $Q$ cavity would render the space charge effect unimportant.) Alterna- tively, the steady state multipactor current at impact ( $I_{m i}=\sigma_{\text {sat }} \mu$, where $\mu$ is constant), relative to the drive current, $I_{d 0}$, is independent of $Q$. Note, however, that in the case of high $Q$, the multipactor current may surge to a very high level transiently before the steady state is reached. ${ }^{8}$ These facts have been verified by computation, as will be explained in Sec. V below. Section V also demonstrates the excellent agreement between this formula [Eq. (5)] and computations. Since we have ignored the space charge effect in the derivation of Eq. (5), we emphasize that Eq. (5) is accurate for $Q>10$. See Table II in Sec. V below for the effects of space charge.

Upon examination of Eq. (5), (at a fixed $v_{0}$ ), we gain insight that the product $s=Q \sigma$ depends on only two external parameters: $r$, the gap voltage amplitude in the absence of multipactor (related to the rf energy stored in the cavity), and $\mu$, the impact velocity at the first crossover point (a function of the wall material). This means that upon determination of those two external parameters, the steady state, if it is reached, is well defined. We can therefore plot the steady state value of $I_{m i} / I_{d 0}=s \mu / r$ vs $\mu$ at different values of $r$ as shown in Fig. 3(a) (for a zero initial velocity). Note that $I_{m i} / I_{d 0}$, the multipactor current relative to the drive current, increases with increasing energy stored in the cavity. Thus the availability of energy favors the multipactor. On the other hand, fixing $r$, the multipactor current (in units of drive current), increases with decreasing final impact velocity $\mu$, up to a point. Beyond that, the multipactor current is expected to drop with a further decrease in $\mu$. However, simulations conducted in that region were unstable and did not saturate. Now remember that the starting impact energy is fixed by the gap voltage (and the geometry) at the start of the multipactor. It seems that if the final impact energy (fixed by the material property $E_{1}$ ) is much below the starting impact energy, then the multipactor becomes unstable and a steady state solution cannot be reached. This will be discussed further in the following section.

Figure 3(b) shows the dependence of the steady state multipactor current on the initial velocity (or emission energy) of secondaries, keeping $r$ and $\mu$ fixed, and assuming $E_{\max }=400 \mathrm{eV}$. Note that there is a velocity that maximizes the multipactor current. However, typical emission velocities lie below that maximum, hence the trend is for increasing $s$ with increasing $v_{0}$. Note that a small change in initial velocity may considerably affect the multipactor. These calculations show the importance of initial emission velocities, as, in reality, there is a wide spread in the emission velocities of the secondary electrons. ${ }^{10,11}$

\section{ACCESSIBILITY OF STEADY STATE MULTIPACTOR}

The above analysis does not say anything about the accessibility of the steady state solution. It simply maps out the steady state multipactor current to the plane spanned by the drive current and by the wall material's first crossover point. If we achieve a steady state at an impact velocity $\mu$ and using a drive current $I_{d 0}$, Eq. (5) then gives the steady state multipactor current expected [see, e.g., Fig. 3(b)]. However, not all steady state solutions are accessible. 

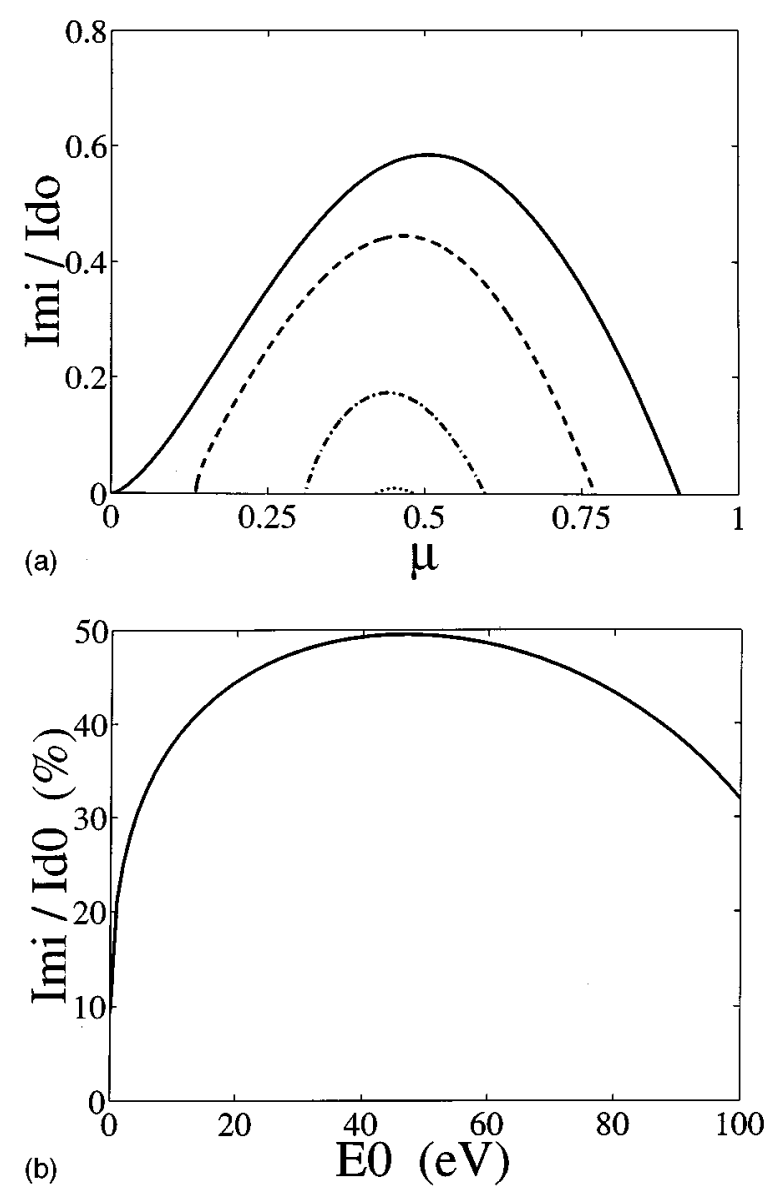

FIG. 3. (a) Steady state multipactor current at impact $I_{m i}$, in units of drive current amplitude $I_{d 0}$, as a function of steady state impact velocity, $\mu=\sqrt{2 E_{1}}$, for different values of empty-cavity steady state gap voltage, $r=Q I_{d 0}$. Legend: $r=0.5(-) ; r=0.4(--) ; r=0.3(-\cdot) ; r=0.27(\cdots)$. (b) Steady state multipactor current at impact $I_{m i}$, in units of drive current amplitude $I_{d 0}$, as a function of emission energy of secondaries, $E_{0}$. Here, $r=0.3$ and $\mu=0.547$ in nondimensional units. The units for $E_{0}$ assume $E_{\text {max }}=400 \mathrm{eV}$. High $E_{0}$ values are included to show the trend.

We know from Vaughan's theory that multipactor can start only if the voltage stays within a narrow range. These voltage boundaries can be derived from the resonance and phase-focusing conditions derived by Vaughan. ${ }^{1,2}$ Vaughan's analysis includes a nonzero monoenergetic emission velocity, $v_{0}$, for the secondaries. The resonance condition, relating the voltage to the launch phase of the electrons, is

$$
V_{g 0}=\frac{\omega^{2}\left(1-N \pi v_{0} / \omega\right)}{2 \sin \theta+N \pi \cos \theta},
$$

where $V_{g 0}$ is the gap voltage amplitude, $\theta$ is the phase of the voltage when the electrons are launched, and $N$ is the order of the multipactor. To maintain the phase resonance, the transit time of the electron should equal an odd number of $\mathrm{rf}$ half cycles, which we call the order $N$ of the multipactor $(N=1,3,5, \ldots)$.

Vaughan has shown that multipactor occurs between $V_{g \text { min }}$, obtained by finding the phase $\theta=\theta_{\min }=\arctan (2 / N \pi)$ that maximizes the denominator of Eq. (6), and $V_{g \text { max }}$, obtained by the requirement that electrons launched (with a general nonzero initial velocity) are able to reach the other plate against a retarding field. Vaughan did not display the formulas for $V_{g \text { min }}$ nor $V_{g \text { max }}$ although he must have used them in the construction of his Fig. 3. ${ }^{1}$ For easy reference, we display, in physical units, $V_{g}$ min in Eq. (7a) for general emission energy $E_{0}$, and $V_{g}$ max in Eq. (7b) only for the special case $E_{0}=0$

$$
\begin{aligned}
& V_{g \min }=\frac{22480(f D)^{2}-(N \pi f D) \sqrt{44960 E_{0}}}{\sqrt{(N \pi)^{2}+4}}, \\
& V_{g \max }=\frac{22480}{N \pi}(f D)^{2},
\end{aligned}
$$

where the voltages are in volts, the frequency $f=\omega / 2 \pi$ is in $\mathrm{GHz}$, the gap width $D$ in $\mathrm{cm}$, and the secondary-electron emission energy $E_{0}$ in $\mathrm{eV}$. There is no simple closed form solution of $V_{g \text { max }}$ for nonzero $E_{0}$.

The discussion in the preceding paragraph means that if the steady state gap voltage, in the presence of multipactor, is higher or lower than the range $\left(V_{g \text { min }}, V_{g \text { max }}\right)$, then multipactor cannot occur. This requires both the starting gap voltage and the final gap voltage to lie within this range, as well as all transients in the gap voltage. Yet, this does not necessarily mean that steady state multipactor is restricted to the range $V_{g \text { min }}<r=Q I_{d 0}<V_{g \text { max }}$. For empty-cavity (no multipactor electrons) steady state gap voltage $r$ below the minimum voltage $V_{g \text { min }}$, multipactor cannot be encountered at all. However, a steady state may still be reached when the empty-cavity steady state gap voltage $r$ is higher than $V_{g \text { max }}$.

The key to achieving such steady states is to examine the transient build up of the gap voltage in the cavity. In the present model, an ideal current source excites the rf cavity with a finite quality factor $Q$, so it takes time for the gap voltage to build up to the steady state level. In this situation, although the steady state voltage in the absence of multipactor can be much higher than the narrow range $\left(V_{g \text { min }}, V_{g \text { max }}\right)$, the gap voltage is bound to transiently pass through it. When that happens, multipactor may start and one of three things will happen. The multipactor current may build up quickly enough to load the cavity and keep the gap voltage within the above narrow range, thus achieving the steady state with a large multipactor current. Alternatively, the voltage may "break-through" the narrow range before the multipactor current has a chance to build up. A third possibility is for the multipactor to build up and load the cavity so rapidly that the voltage is reduced below $V_{g}$ min , at which point the multipactor quenches. These types of behavior are typically observed in the course of operation of various accelerator structures (see Fig. 4). ${ }^{12}$

What determines whether the voltage breaks through the multipactor region, $\left(V_{g \text { min }}, V_{g \text { max }}\right)$, or whether it stays within this region allowing a steady state multipactor? Two processes are competing in this scenario, each characterized by a different parameter: (1) The voltage in the cavity building up, with a time constant proportional to $Q$; (2) the multipactor current building up, at a rate dependent upon the slope of the secondary electron yield curve (Fig. 2). If the rates are comparable, then a steady state multipactor can be achieved. Typically this occurs for a high $Q$ structure $(\sim 1000)$. The 


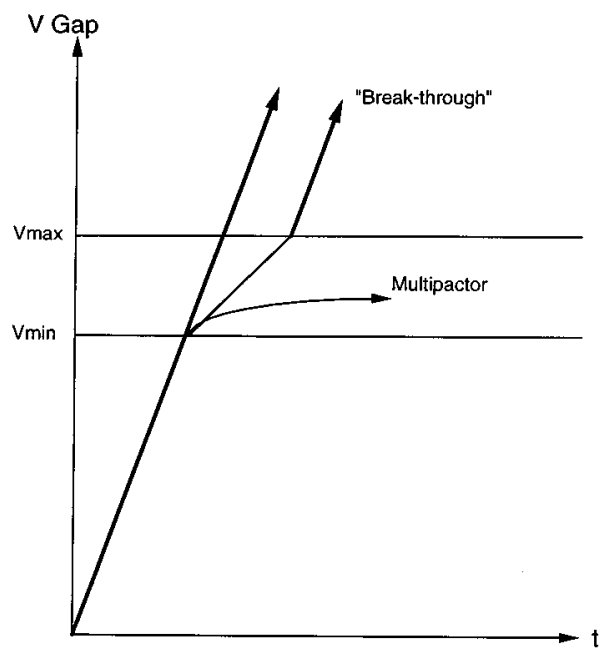

Representation of gap voltage variation with time

FIG. 4. Representation of gap voltage variation with time (Ref. 12).

latter situation is a very delicate balance between the rate of energy storage in the cavity and the rate of buildup of multipactor current.

Figure 5 shows the results of a simulation exhibiting such a steady state multipactor. With $Q=1000, r=Q I_{d 0}$ was chosen to be 0.5 , somewhat higher than $V_{g \text { max }}=0.32$ (in the same nondimensional units) for a first-order multipactor with $v_{0}=0$. However, as Fig. 5(a) displays, a steady-state multipactor can be achieved, and at quite a high level, too. The steady state level of $I_{m i} / I_{d 0}=54 \%$ agrees well with what Eq. (5) predicts. This enormous level of steady state multipactor current (more than half the drive current) is not surprising. A glance at Fig. 3(a) shows that the steady state charge density (or multipactor current) increases rapidly with increasing $r$. This large current loads the cavity quite a bit, holding the voltage to within the multipactor region where it started. Figure 5(b) shows the time behavior of the voltage, where the dotted lines outline the multipactor region $\left(V_{g \text { min }}=0.268\right.$; $V_{g \text { max }}=0.318$ ). Initially the multipactor current is low and the voltage slowly grows in response to the drive current. As the multipactor current grows, however, it loads the cavity and pins the voltage down. Figure 5(c) displays the secondary electron yield at times of impact, indicating it hovers around unity.

If $Q$ is low while the multipactor is growing slowly, breakthrough will be achieved instead. When we lowered $Q$ to 10 (instead of 1000 as in Fig. 5), the voltage rapidly rose above the multipactor region (Fig. 6), where the phase resonance was lost. Eventually, the electric field had the phase to push the newly emerging secondaries back to their birthplace, quenching the multipactor. In such cases, the rise time of the gap voltage is so short that the multipactor current does not have a chance to build up to a level sufficient to load the cavity.

On the other hand, combining a high multipactor growth rate (e.g., using a material with large $\delta_{\text {max }}$ or low $E_{1}$ ) with a high $Q$ (hence a slowly changing voltage) allows the multipactor current to grow to such large values without the volt-
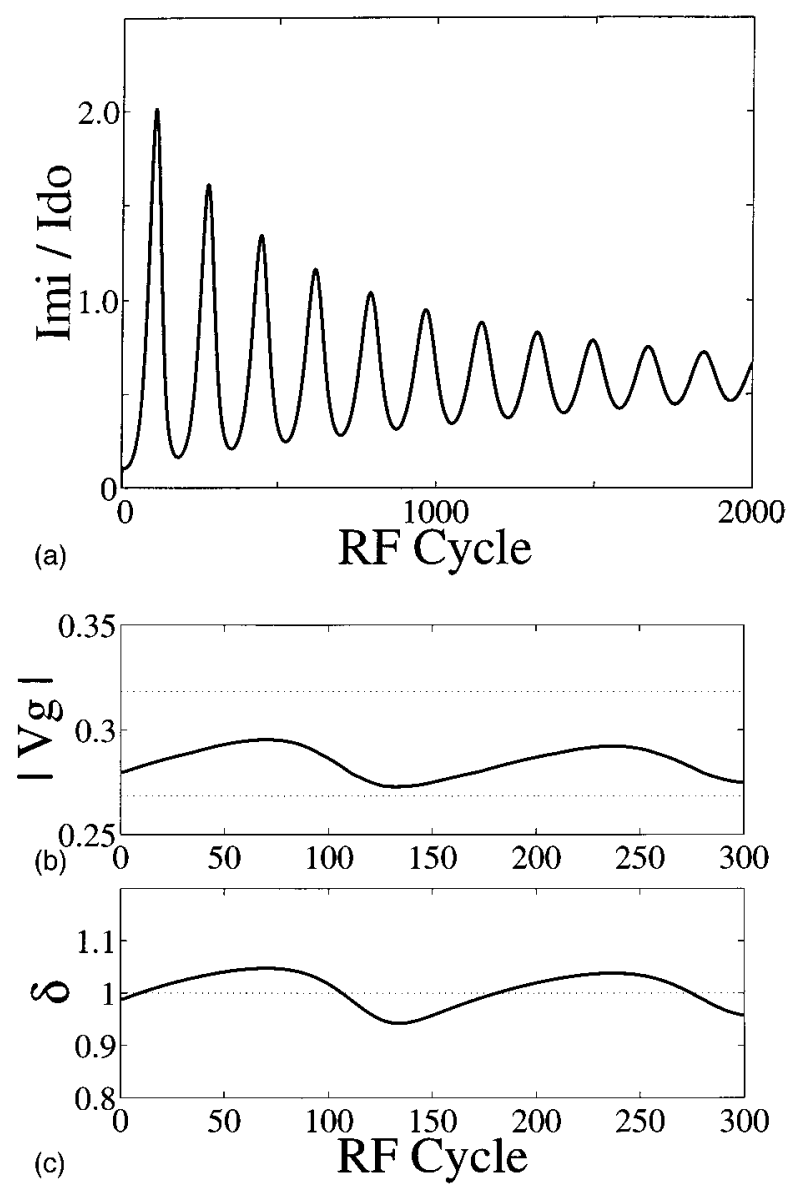

FIG. 5. (a) Transient evolution of multipactor current, in units of drive current amplitude $I_{d 0}$, for $r=Q I_{d 0}=0.5$ and $Q=1000 \quad\left(E_{0}=0\right.$ and $\mu=0.547$ ). (b) Transient evolution (on a shorter time scale) and the (nondimensional) gap voltage amplitude, for $r=Q I_{d 0}=0.5$ and $Q=1000$. The dotted lines at $V_{g 0}=0.268$ and $V_{g 0}=0.318$ outline the limits of the multipactor region. (c) Transient evolution of the secondary electron yield for $r=Q I_{d 0}=0.5$ and $Q=1000$.

age moving rapidly enough to its steady state value. In this case, the multipactor current reached may be sufficient to load the gap voltage down to below $V_{g \text { min }}$, hence upsetting its own phase resonance requirement, at which point it will

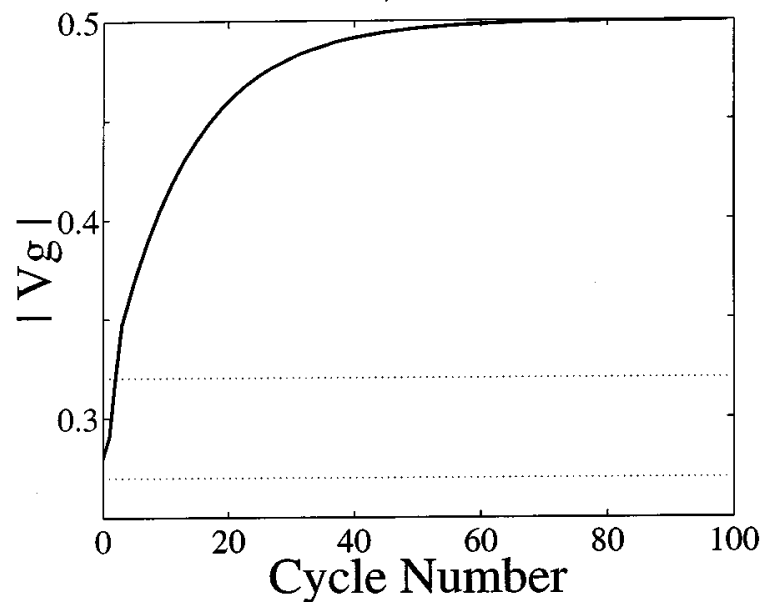

FIG. 6. Transient evolution of (nondimensional) gap voltage amplitude, for $r=Q I_{d 0}=0.5$ and $Q=10 \quad\left(E_{0}=0\right.$ and $\left.\mu=0.547\right)$. The dotted lines at $V_{g 0}=0.268$ and $V_{g 0}=0.318$ outline the limits of the multipactor region. 


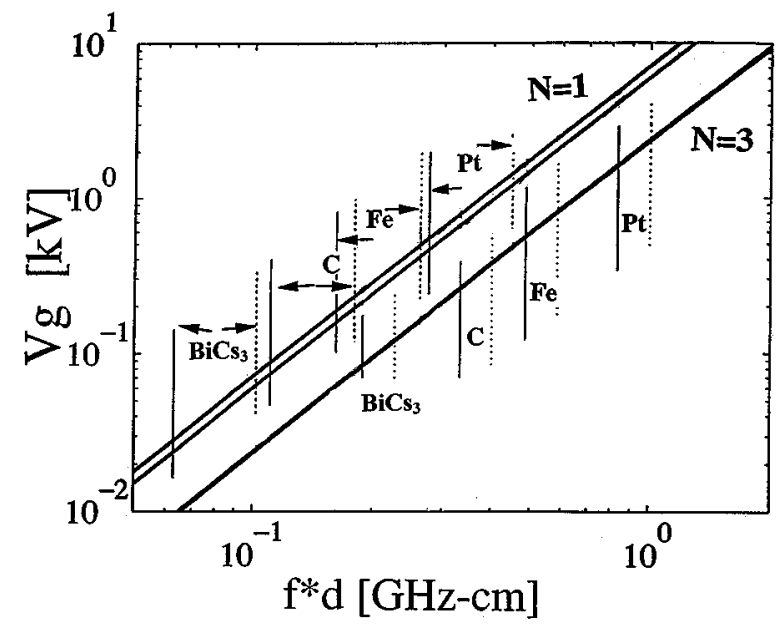

FIG. 7. Susceptibility curve showing the regions of $\left(V_{g 0}, f D\right)$ space prone to first and third order multipactors, for four materials: $\mathrm{BiCs}_{3}, \mathrm{C}$ (diamond), $\mathrm{Fe}$, and Pt. The secondary electrons are assumed to be emitted with zero velocities.

drift in phase (perhaps fluctuate to higher order multipactors) and eventually die out once it encounters the wrong phase of rf. ${ }^{13}$ When that happens, the voltage will build up again to a value above $V_{g} \min$, and the whole scenario may repeat again and again. Physically, the multipactor will appear as a series of random "spikes" that appear sporadically, grow, and then disappear.

In the above analysis we saw how important the effect of the wall material is in determining the behavior of a multipactor. For a given material with a given secondary electron emission curve, we can further narrow down the range of accessible solutions. The underlying reason is that the yield has to be above unity for a discharge to occur, and the impact energy has to be in the vicinity of the first crossover point. If the impact energy of the electrons is much higher than $E_{1}$, even though the yield may be above 1, the multipactor current will grow to such large values before sufficiently loading the cavity to the steady state that it will destroy its own phase resonance, a scenario described in the preceding paragraph (see also Ref. 13). In that case, the multipactor will be observed in bursts, and a steady state is not achieved. Therefore, only if the voltage is made to sweep quickly through the multipactor-prone region, multipactor will have a smaller chance to grow to saturation before breaking through. ${ }^{12}$

The boundaries described above can be translated onto a plot of voltage versus the geometry factor $(f D)$. Traditionally such plots are referred to as susceptibility curves, and define the boundaries of regions prone to $N$ th order multipactor. Since the voltage scales as the square of this factor for zero emission velocity [cf. Eq. (7)], the boundaries $V_{g \text { min }}$ and $V_{g \text { max }}$ can be represented as straight lines with a slope of 2 on a $\log -\log$ plot (Fig. 7). As discussed in the preceeding paragraph, the starting impact energy cannot be below $E_{1}$ (or else $\delta<1$ ), and cannot exceed $E_{1}$ by much. Hence one boundary is that the maximum possible impact energy must be greater than $E_{1}$. The second boundary on the impact energy is not immediately obvious and it depends on a lot of parameters. Our extensive simulations (with $Q$ in the
TABLE I. Material data (Ref. 14).

\begin{tabular}{cccc}
\hline \hline Material & $\delta_{\max }$ & $E_{\max }(\mathrm{eV})$ & $E_{1}(\mathrm{eV})$ \\
\hline $\mathrm{BiCs}_{3}$ & 6.5 & 1000 & 18 \\
$\mathrm{C}$ (diamond) & 2.8 & 750 & 56 \\
$\mathrm{Fe}$ & 1.3 & 400 & 120 \\
$\mathrm{Pt}$ & 1.5 & 750 & 350 \\
\hline \hline
\end{tabular}

range $10-100$ and $\delta_{\max }=1.2$ ) show that a steady state multipactor cannot be reached if the minimum possible starting impact energy is greater than $1.33 E_{1}$, so we will use this as the second boundary. We emphasize, however, that this boundary is best estimated by simulations that are run with data of the actual conditions.

Ignoring any nonzero initial velocity of secondaries, the impact energy can be related to the driving voltage (in the absence of multipactor) by solving the force law to give

$$
E_{i}=\frac{2 V_{g 0}^{2}}{\omega^{2}} \cos ^{2} \theta
$$

The maximum possible impact energy, $E_{i \text { max }}$, can be solved by setting $V_{g 0}=V_{g \text { max }}$ and $\theta=0$. Setting that equal to $E_{1}$ gives the first boundary, the solid vertical line on the susceptibility curve (see Fig. 7). Similarly, using $V_{g} \min$ and $\theta_{\min }$ in Eq. (8) results in the minimum possible impact energy, $E_{i \min }$. Setting $E_{i \min }$ to $1.33 E_{1}$ gives the second boundary, which we represent with the dotted vertical line in Fig. 7. Including the scaling factors, the solid and dotted vertical lines in Fig. 7 define the interval

$$
0.1483 \cdot N \sqrt{\frac{E_{1}}{100}}<f D<0.171\left(N+\frac{4}{N \pi^{2}}\right) \sqrt{\frac{E_{1}}{100}},
$$

where $f$ is in $\mathrm{GHz}, D$ in $\mathrm{cm}$, and $E_{1}$ in $\mathrm{eV}$.

Figure 7 represents the susceptibility curves obtained in this manner for a host of representative materials for a first and a third order multipactors, assuming a zero emission velocity of the secondary electrons. (The two curves for $V_{g \text { min }}$ and $V_{g \text { max }}$ for $N=3$ are so close that they appear as one line on the scale of Fig. 7.) Table I lists the secondary emission properties of these materials. Vaughan's empirical formula ${ }^{11}$ has been used in calculating $E_{1}$ from $\delta_{\max }$ and $E_{\max }$ in this table. These materials are chosen only to illustrate the concept, particularly since their susceptibility regions are well separated in Fig. 7. Note from that figure that the higher order multipactors occur for higher $(f D)$ products as well as higher voltages. These susceptibility curves, derived from a dynamic theory, show the qualitative features of the experimentally observed data, as will be shown in Sec. V.

\section{COMPARISON OF THE STEADY STATE AND ACCESSIBILITY THEORIES TO SIMULATION AND TO PUBLISHED DATA}

We ran several representative simulations (in the region that allows a steady state solution) to test the theory for the steady state. Tables II-IV below illustrate the excellent agreement between theory and simulation. The simulations 
TABLE II. Comparison of theory and simulations including image space charge force $(r=0.3 ; \mu=0.547)$.

\begin{tabular}{crccccc}
\hline \hline & & $s$ & \multicolumn{2}{c}{$s$ (Simulations) } & $I_{m i} / I_{d 0}$ & $(\%)$ \\
\multicolumn{1}{c}{$I_{d 0}$} & \multicolumn{1}{c}{$Q$} & (Theory) & $(\mathrm{BL})$ & $(\mathrm{BL}+\mathrm{SC})$ & $(\mathrm{BL})$ & $(\mathrm{BL}+\mathrm{SC})$ \\
\hline 0.3000 & 1 & 0.051 & 0.051 & 0.025 & 9.2 & 4.5 \\
0.0300 & 10 & 0.051 & 0.051 & 0.046 & 9.2 & 8.4 \\
0.0030 & 100 & 0.051 & 0.051 & 0.050 & 9.2 & 9.1 \\
0.0003 & 1000 & 0.051 & 0.051 & 0.051 & 9.2 & 9.2 \\
\hline \hline
\end{tabular}

in Table II were done with $E_{0}=0, r=Q I_{d 0}=0.3$, and $\mu=0.547$. For a frequency of $1 \mathrm{GHz}$ and a gap width of 0.22 $\mathrm{cm}$, these values correspond to an empty-cavity gap voltage amplitude of $330 \mathrm{~V}$ and a first cross-over energy of $166 \mathrm{eV}$. In Table II, "theory" refers to the steady state value predicted by the analytic formula (5), "simulation" refers to the numerical integration of Eqs. 1-3, "BL' refers to beam loading only [i.e., space charge forces are ignored as in Eq. (3)], and "BL $+\mathrm{SC}$ " refers to the inclusion of image space charge force through the addition of the term $\sigma(x-1 / 2)$ to the right hand side of Eq. (3). ${ }^{8,9}$ Table II indicates that image space charge forces are not quite important in determining the saturation level of the multipactor current, especially for high $Q$ cavities. Note that, ignoring the effect of these space charge forces, the multipactor current relative to the drive current is independent of $Q$.

Tables III and IV illustrate agreement between theory [Eq. (5)] and simulations for different combinations of the external parameters $r, \mu$, and $v_{0}$. Space charge effects are ignored in Tables III and IV. (In the three cases marked by an asterisk in Table III, the simulations include space charge effects.) In Table III, we set the secondaries' initial energy $E_{0}$ equal to zero. In Table IV, the effects of nonzero initial energy are displayed. Note the general trend for stronger multipactor currents (higher $s$ ) with higher drive currents (high $r$ ), lower impact energies (low $\mu$ ), or higher initial velocities (high $v_{0}$ ), as discussed in Sec. III above.

Figure 8 maps out the simulations of Table III in $(r, \mu)$ space. The two horizontal lines mark $V_{g \text { min }}$ and $V_{g \text { max }}$, while the two vertical lines outline the region of final impact velocities for which steady state solutions are accessible (see Sec. IV). Steady state solutions have been observed for all points located within those four lines. For points above
TABLE IV. Comparison of theory and simulations for various initial energies $E_{0}(r=0.3 ; \mu=0.547 ; Q=10)$.

\begin{tabular}{cccc}
\hline \hline $\begin{array}{c}E_{0} \\
(\mathrm{eV})\end{array}$ & $\begin{array}{c}s \\
\text { (Theory) }\end{array}$ & $\begin{array}{c}s \\
\text { (Simulation) }\end{array}$ & $\begin{array}{c}I_{m i} / I_{d 0} \\
(\%)\end{array}$ \\
\hline 0 & 0.051 & 0.051 & 9.2 \\
2 & 0.138 & 0.138 & 25.2 \\
5 & 0.175 & 0.175 & 31.9 \\
10 & 0.209 & 0.209 & 38.1 \\
20 & 0.247 & 0.244 & 44.5 \\
\hline \hline
\end{tabular}

$V_{g \text { max }}$, such as that one point at $(0547,0.5)$, a steady state has been observed only under certain conditions (such as very high $Q$ ), as explained in Sec. IV. For points to the right of the solid vertical line, no multipactor has been observed because the secondary electron yield is always less than unity. Finally, although we have observed multipactor to the left of the dotted vertical line, we have not yet found any simulations that reach a steady state in that area. Note that for the simulations where we have observed a steady state, the multipactor current increases in the direction indicated by the arrow on Fig. 8.

This trend is also displayed graphically in Figs. 9 through 11. In these figures, the multipactor current is displayed as function of time for a set of different external parameters. In Fig. 9, $r$ is changed, whereas in Fig. 10, $\mu$ is changed, and in Fig. 11, $v_{0}$ is changed. The steady state behavior compares well with the theory of Sec. III. Examining the time evolution of the discharge (Figs. 9, 10), we can see that, in general, a larger steady state multipactor current (relative to drive current) forces a faster response for cavities with the same $Q$. (Of course, response time is proportional to $Q$, so a high $Q$ cavity responds more slowly.) One exception, however, is evident from Fig. 11. Even though different initial velocities of secondaries result in different steady state multipactor currents, the rise time is the same regardless of $v_{0}$ !

The susceptibility curves similar to those derived in Sec. IV have been used extensively in the literature. ${ }^{1,6,15-18}$ For example, experiments conducted by Hatch and Williams in 1950 's outlined similar regions in ( $f D$ vs gap voltage) space where multipactor breakdown was observed. ${ }^{15,16}$ One such

TABLE III. Comparison of theory and simulations $\left(E_{0}=0\right)$.

\begin{tabular}{lcccccr}
\hline \hline & & $r$ & & $s$ & $s$ \\
$I_{d 0}$ & $Q$ & $\left(=I d 0^{*} Q\right)$ & $\mu$ & (Theory) & (Simulation) & $\begin{array}{c}I_{m i} / I_{d 0} \\
(\%)\end{array}$ \\
\hline 0.0285 & 10 & 0.285 & 0.547 & 0.008 & $0.007^{*}$ & 1.5 \\
0.0300 & 10 & 0.300 & 0.547 & 0.051 & 0.051 & 9.2 \\
0.0310 & 10 & 0.310 & 0.547 & 0.078 & $0.071^{*}$ & 13.8 \\
0.0300 & 10 & 0.300 & 0.554 & 0.044 & 0.044 & 8.1 \\
0.0300 & 10 & 0.300 & 0.547 & 0.051 & 0.051 & 9.2 \\
0.0300 & 10 & 0.300 & 0.539 & 0.058 & 0.058 & 10.3 \\
0.0310 & 10 & 0.310 & 0.547 & 0.078 & $0.071^{*}$ & 13.8 \\
0.0310 & 10 & 0.310 & 0.529 & 0.094 & 0.094 & 16.1 \\
0.0310 & 10 & 0.310 & 0.510 & 0.110 & 0.110 & 18.1 \\
0.0003 & 1000 & 0.300 & 0.547 & 0.051 & 0.051 & 9.2 \\
\hline \hline
\end{tabular}




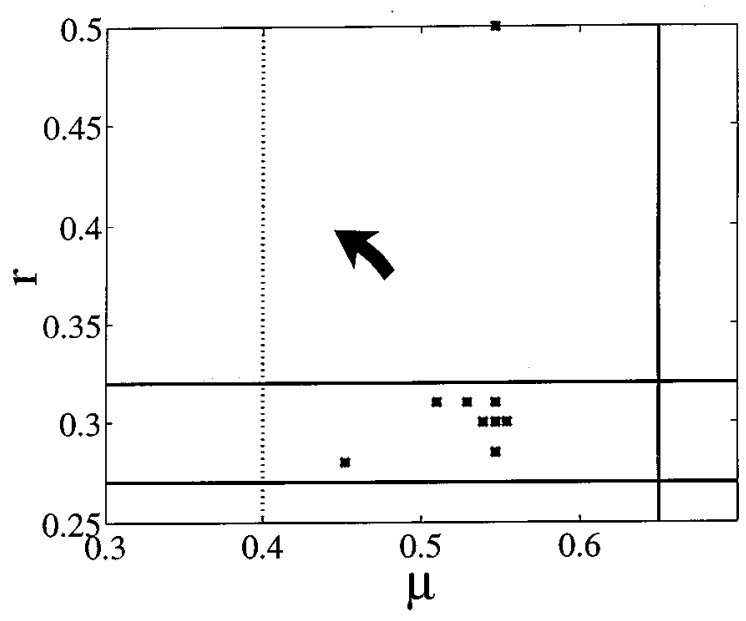

FIG. 8. Location in $(r, \mu)$ space of simulations displayed in Table III. Multipactor current increases in the direction of the arrow.

region is shown in Fig. 12, redrawn from Ref. 15. Note the two well-defined diagonal lines, and the lower vertical cutoff line (cf. Fig. 7). Their experimental apparatus was unable to reach the region of the upper cutoff. To explain these experimental curves, Hatch and Williams use a complicated theory called constant $k$ theory, based on an ad hoc assumption that a certain parameter, $k$, equal to the ratio of the impact velocity to the initial velocity of secondaries, is constant. The "constant" value of this parameter is obtained by fitting the results of the theory to the experimental curves. Our theory, on the other hand, is deductive, but the fundamental limitation lies with the assumption of a monoenergetic velocity, so it is difficult to compare to existing experimental data with unknown initial velocity profiles.

\section{CONCLUSION}

In this paper, we used the premise that saturation of a multipactor occurs primarily due to beam-loading effects, at an impact energy near the first crossover point in the second-

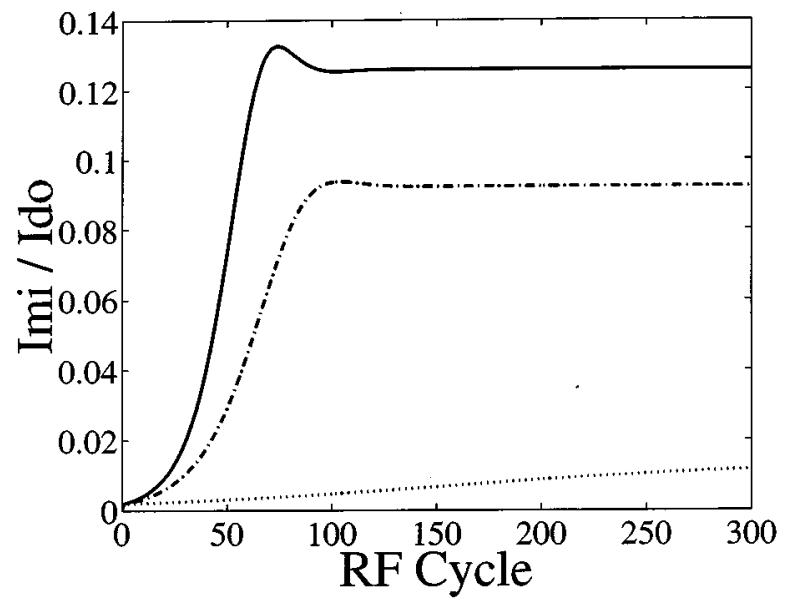

FIG. 9. Transient evolution of multipactor current, in units of drive current amplitude $I_{d 0}$, for various values of $r=Q I_{d 0}$. Here, $Q=10, v_{0}=0$ and $\mu=0.547$. Legend: $r=0.31(-) ; r=0.3(-\cdot-) ; r=0.285(\cdots)$.

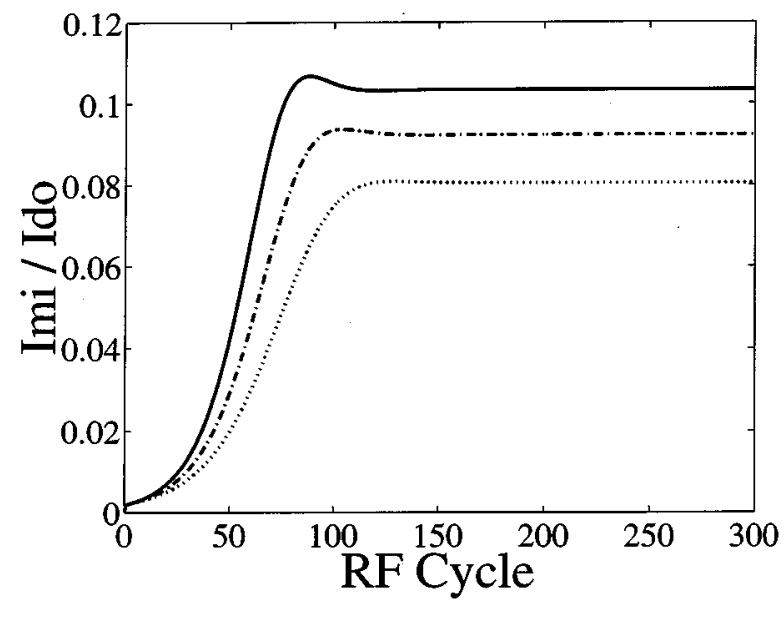

FIG. 10. Transient evolution of multipactor current, in units of drive current amplitude $I_{d 0}$, for various values of $\mu=\sqrt{2 E_{1}}$. Here, $Q=10, v_{0}=0$ and $r=0.3$. Legend: $\mu=0.539$ (一); $\mu=0.547(-\cdot-) ; \mu=0.554(\cdots)$.

ary electron yield curve, to derive the steady state level of the multipactor current. This level depends on three external parameters, namely, the energy supplied by the cavity, the first crossover energy and the emission energy of the (assumed monoenergetic) secondaries. These levels have been very favorably compared with simulations.

Further, the accessibility of such steady state solutions have been investigated. It has been shown that steady state multipactor can occur while the cavity is powered up, when the (transient) voltage passes through the multipactor region, even though the steady state voltage of the multipactor-free cavity is much higher than this range. Various scenarios of the transient evolution are addressed when steady states do not occur. These considerations lead to a set of susceptibility curves which determine whether a steady state multipactor can be attained for various materials. The assumption of monoenergetic emission velocity, and the rather sensitive dependence on it (e.g., in the multipactor saturation level, or in the susceptibility region boundaries) makes a meaningful

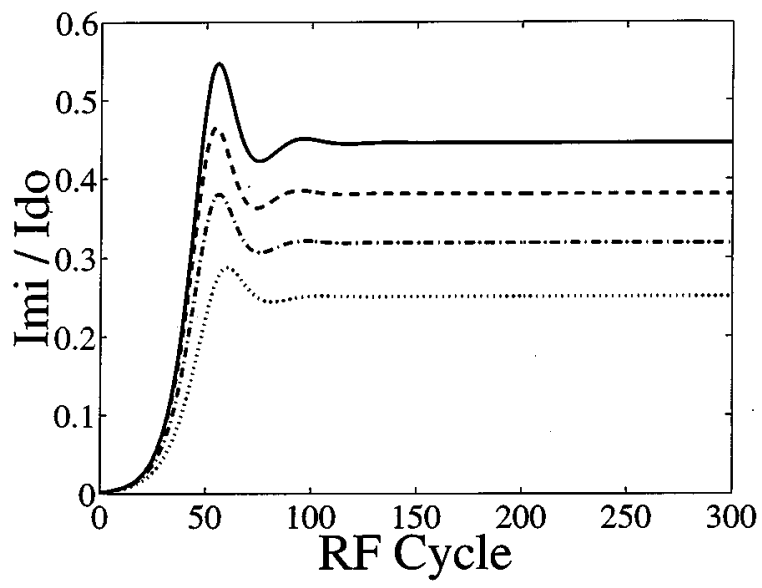

FIG. 11. Transient evolution of multipactor current, in units of drive current amplitude $I_{d 0}$, for various values of $E_{0}$. Here, $Q=10, r=0.3$ and $\mu=0.547$. For $E_{\max }=400 \mathrm{eV}, E_{0}=20 \mathrm{eV}(-) ; E_{0}=10 \mathrm{eV}(--) ; E_{0}=5 \mathrm{eV}(--) ; E_{0}=2$ $\mathrm{eV}(\cdots)$. 


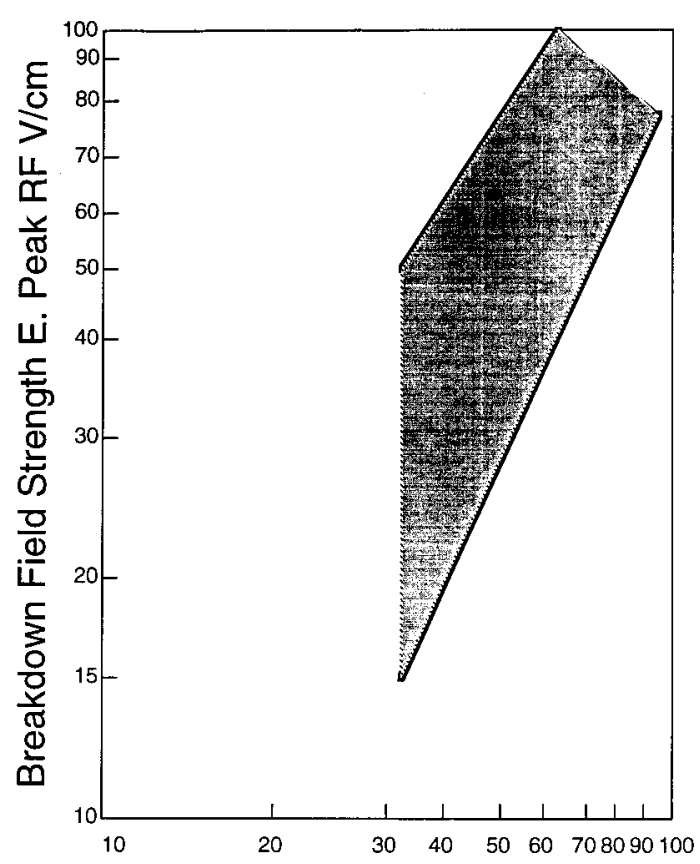

Frequency in Megacycles

FIG. 12. Experimental susceptibility curve measured for alclad electrodes $\left(E_{1}=53 \mathrm{eV}\right)$ with a gap separation of $3 \mathrm{~cm}$ (Ref. 15).

comparison of multipactor data contingent upon the availability of the emission velocity distribution, and a numerical simulation incorporating such a distribution.

\section{ACKNOWLEDGMENTS}

We wish to acknowledge numerous useful discussions with Dr. Spilios Riyopoulos, Dr. Carter Armstrong, and Professor Ronald Gilgenbach. We further wish to thank Dr. Venkatesh Gopinath, Dr. John Verboncoeur, and Professor Charles (Ned) K. Birdsall, of the University of California at Berkeley for their interest in our work.

This work was supported by the Naval Research Laboratory/Office of Naval Research, by the Naval Surface Warfare Center/Crane Division, and by the Multidisciplinary University Research Initiative (MURI), managed by the Air Force Office of Scientific Research and subcontracted through Texas Tech University.

\section{APPENDIX: DERIVATION OF THE STEADY STATE MULTIPACTOR}

In this appendix, we demonstrate the solution of the steady state multipactor equations using Fourier series, for the case of a first-order, two-surface, multipactor with no space charge forces and driven at resonance $(\omega=1)$. Since Eqs. (1) and (2) are valid only between impacts, i.e., $0<t<t_{i}=\pi$, we need to extend them to periodic functions that are valid for all time in order to use Fourier series. Since the multipactor current is proportional to the velocity of the sheet, and since this velocity has a discontinuity (step function) at impact, changing from $\mu=\sqrt{2 E_{1}}$ (impact velocity of primaries) to $-v_{0}$ (emission velocity of secondaries in the opposite direction of the impacting primaries), the derivative of the multipactor current in Eq. (1) can be represented as the sum of Dirac $\delta$ functions at the points of impact. This results in

$$
\begin{aligned}
\left(\frac{d^{2}}{d t^{2}}+\right. & \left.\frac{1}{Q} \frac{d}{d t}+1+\sigma\right) V_{g}(t) \\
= & \frac{d}{d t} I_{d}(t)+\sigma\left(\mu+v_{0}\right) \sum_{m}\left[u_{0}(t-2 m \pi)\right. \\
& \left.-u_{0}(t-(2 m+1) \pi)\right] .
\end{aligned}
$$

In this appendix, $\sigma$ means $\sigma_{\text {sat }}$, the steady state charge density (constant, and positive by convention), while $I_{d}(t)=I_{d 0} \sin (\omega t+\phi), \phi$ being the relative phase between the multipacting sheet and the rf drive at the steady state, and $u_{0}$ is the Dirac $\delta$ function. The summation index extends to all integers: positive, zero, and negative.

Since the gap voltage is periodic with period $2 \pi$, it can be expressed as a Fourier series

$$
V_{g}=\sum_{n} V_{n} e^{j n t}
$$

Using Euler's relation to decompose the drive current, $I_{d}(t)$, into its two Fourier components $(n= \pm 1)$, and the fact that

$$
\sum_{m} u_{0}(t-2 m \pi)=\frac{1}{2 \pi} \sum_{n} e^{j n t},
$$

Eq. (A1) can be solved to determine the Fourier coefficients of the voltage

$$
\begin{aligned}
& V_{n}=\frac{j n I_{d n}+\sigma\left(\mu+v_{0}\right) / \pi}{\left(-n^{2}+j n / Q+1+\sigma\right)}, \quad n \text { odd, } \\
& V_{n}=0, \quad n \text { even. }
\end{aligned}
$$

Observing that the voltage is almost sinusoidal, we can neglect higher order terms $(|n|>1)$. This can be justified by examining the denominator on the right hand side of (A3). For $n=1$, the denominator becomes equal to $j / Q+\sigma$. Recognizing that the product $\sigma Q \ll 1$, we know that this denominator is small relative to the numerator, and hence the contribution of the $n=1$ term is significant. As we shall see, the next higher term, $n=3$, would contribute a quantity about 30 times smaller than the $n=1$ term.

The two unknowns, $\sigma$ and $\phi$, can be found by imposing the two steady state conditions, derived by integrating the force law, Eq. (3), once,

$$
\begin{aligned}
\int_{0}^{\pi} V(t) d t=\left.\dot{x}\right|_{t=\pi}-\left.\dot{x}\right|_{t=0} & \Rightarrow \int_{0}^{\pi} \sum_{n} V_{n} e^{j n t} d t \\
& =\sum_{n} V_{n} \int_{0}^{\pi} e^{j n t} d t=\mu-v_{0},
\end{aligned}
$$

and then again, using integration by parts to simplify the resulting double integral, 


$$
\begin{aligned}
\int_{0}^{\pi}(\pi-\tau) V(\tau) d \tau+\int_{0}^{\pi} v_{0} d \tau & =\left.x\right|_{t=\pi} \\
& \Rightarrow \int_{0}^{\pi}(\pi-\tau) \sum_{n} V_{n} e^{j n \tau} d \tau \\
& =\sum_{n} V_{n} \int_{0}^{\pi}(\pi-\tau) e^{j n \tau} d \tau \\
& =1-v_{0} \pi .
\end{aligned}
$$

Note that a nonzero initial velocity affects the solution by reducing the effective steady state impact energy and by reducing the effective gap width.

Note that, from (A3), $V_{n} \propto 1 / n^{2}$ for large $n$. The infinite sums in (A4) and (A5) decay as $1 / n^{3}$ for large $n$. Thus in the infinite sums in both (A4) and (A5), we need only to keep the $n= \pm 1$ terms. In so doing, we have two equations in two unknowns, $\sigma$ and $\phi$, and the quality factor $Q$ appears only in combinations with the driver current magnitude, $I_{d 0}$, or with the charge density, $\sigma$. In other words, there is no explicit dependence on $Q$, as explained in Sec. III. Defining $r \equiv Q I_{d 0}$ and $s \equiv Q \sigma_{\text {sat }}$, we can rewrite Eqs. (A4) and (A5) by taking only the $n= \pm 1$ terms in the infinite sums

$$
\mu-v_{0}=\frac{2}{1+s^{2}}\left[r \cos \phi-s r \sin \phi-\frac{2\left(\mu+v_{0}\right) s}{\pi}\right],
$$

and

$$
\begin{aligned}
1-v_{0} \pi= & \frac{1}{1+s^{2}}\left[2 r \sin \phi+\pi r \cos \phi-2\left(\mu+v_{0}\right) s\right. \\
& \left.-\pi s r \sin \phi+2 s r \cos \phi-\frac{4\left(\mu+v_{0}\right) s^{2}}{\pi}\right] .
\end{aligned}
$$

Finally, $\phi$ can be eliminated from Eqs. (A6) and (A7) to give the result in Eq. (5).
${ }^{1}$ J. R. M. Vaughan, IEEE Trans. Electron Devices ED-35, 1172 (1988).

${ }^{2}$ S. Riyopoulos, D. Chernin, and D. Dialetis, Phys. Plasmas 2, 3194 (1995).

${ }^{3} \mathrm{~F}$. Mako and W. Peter, IEEE Proceedings of the 1993 Particle Accelerator Conference (Institute of Electrical and Electronics Engineers, Piscataway, NJ, 1993) [IEEE Cat. No. 93CH3279-7], p. 2702.

${ }^{4}$ A. S. Gilmore, Microwave Tubes (Artech House, Norwood, MA, 1986), p. 474.

${ }^{5}$ G. A. Loew and J. W. Wang, Proceedings of the 1989 Particle Accelerator Conference (Institute of Electrical and Electronics Engineers, Picataway, NJ, 1989) [IEEE Cat. No. 89CH2669-0], p. 1137; K. J. Kleman, Proceedings of the 1993 Particle Accelerator Conference (Institute of Electronics and Electrical Engineers, Piscataway, NJ, 1993) [IEEE Cat. No. 93CH3279-7], p. 924.

${ }^{6}$ A. D. Woode and J. Petit, Microwave J. January, 142, (1992).

${ }^{7}$ J. R. M. Vaughan, IEEE Trans. Electron Devices ED-15, 883 (1968).

${ }^{8}$ R. Kishek and Y. Y. Lau, Phys. Rev. Lett. 75, 1218 (1995).

${ }^{9}$ R. Kishek and Y. Y. Lau, Phys. Plasmas 3, 1481 (1996). The phasefocusing mechanism unearthed in this reference may result in a very narrow bunch despite the mutual repulsion among the space charges. Hence we ignore the electrostatic repulsion here. Electrostatic repulsion among the space charges was studied in Refs. 1-3 and 19.

${ }^{10} \mathrm{~A}$ distribution in the initial velocity of secondaries requires particle simulations in general. Analysis of initial velocity distributions have been studied recently by Riyopoulos et al., in Ref. 19 .

${ }^{11}$ J. R. M. Vaughan, IEEE Trans. Electron Devices ED-36, 1963 (1989); A. Shih and C. Hor, IEEE Trans. Electron Devices ED-40, 824 (1993); C. K. Birdsall and W. B. Bridges, Electron Dynamics of Diode Regions (Academic, New York, 1966).

${ }^{12}$ Linear Accelerators, edited by P. M. Lapostolle and A. L. Septier (NorthHolland, Amsterdam 1970), p. 917.

${ }^{13}$ If the transient multipactor current builds up to very high values, space charge effects will set in and may cause saturation, as investigated by Riyopoulos et al., in Ref. 19.

${ }^{14}$ O. Hachenberg and W. Brauer, Adv. Electron. Electron Phys. XI, 413 (1959).

${ }^{15}$ A. J. Hatch and H. B. Williams, J. Appl. Phys. 25, 417 (1954).

${ }^{16}$ A. J. Hatch and H. B. Williams, Phys. Rev. 112, 681 (1958).

${ }^{17}$ P. F. Clancy, Microwave J., March, 77 (1978).

${ }^{18}$ N. Rozario, H. F. Lenzig, K. F. Reardon, M. S. Zarro, and C. G. Baran, IEEE Trans. Microwave Theory Technol. MTT-42, 558 (1994).

${ }^{19}$ S. Riyopoulos, D. Chernin, and D. Dialetis, "Effect of random secondary delay times and emission velocities in electron multipactor," to appear in IEEE Trans. Electron Devices (1997). 